\title{
Predicting bone loss following orthotopic liver transplantation
}

\author{
O M Crosbie, R Freaney, M J McKenna, M P Curry, J E Hegarty
}

\begin{abstract}
Background-Hepatic osteodystrophy occurs in the majority of patients with advanced chronic liver disease with the abnormalities in bone metabolism accelerating following orthotopic liver transplantation (OLT).

Aims-To examine changes in bone mineral density (BMD) following OLT and to investigate factors that lead to bone loss. Methods-Twelve patients had BMD (at both the lumbar spine (LS) and femoral neck (FN)) and biochemical markers measured preoperatively and for 24 months following OLT.

Results-BMD was low in $75 \%$ of patients prior to OLT and decreased significantly from baseline at the LS at three months and the FN at six months. BMD began to increase thereafter at both sites, approaching baseline values at the LS by 12 months. Bone formation markers, osteocalcin and procollagen type I carboxy propeptide, decreased immediately postOLT, with a concomitant increase seen in the resorption markers pyridinoline and deoxypyridinoline. This resulted in a negative uncoupling index early postOLT, that rebounded to positive values after six months. There was a significant correlation between the change in the uncoupling index between six and three months which preceded the increase in BMD at 12 months. The decrease in BMD recorded early post-OLT correlated with vitamin $D$ levels at three months.

Conclusions-Results suggest that increased resorption and inadequate formation are the major contributors to additional bone loss following OLT. Noninvasive biochemical markers precede later changes in BMD in this patient group following OLT and may have a role in investigating and planning intervention strategies to prevent bone loss in future studies.

(Gut 1999;44:430-434)
\end{abstract}

Keywords: liver transplantation; bone mineral density; bone resorption

Hepatic osteodystrophy occurs in up to $50 \%$ of patients with chronic liver disease and is mainly due to an imbalance between bone formation and bone resorption that results in osteoporosis. ${ }^{1-4}$ Previous studies using biochemical markers of bone formation and resorption have shown that increased resorption is the major contributor to the development of hepatic osteodystrophy. ${ }^{1}$ Further bone loss occurs in the first few months following orthotopic liver transplantation (OLT) and results in fracture rates of $17-65 \%$ in patients in the first year following OLT. ${ }^{5-9}$ Serial bone mineral density (BMD) measurements performed after OLT show that the greatest loss in bone occurs in the first three to six months. ${ }^{510} \mathrm{BMD}$ is regained by the end of the first year reaching levels close to preoperative values, increases above baseline values at two years, and continues to increase for up to five years. ${ }^{681112}$

No postoperative clinical parameters identify early those patients most at risk of bone loss following OLT, ${ }^{10111314}$ but a low BMD preoperatively seems to predispose to fractures postoperatively. ${ }^{8}$ If clinical and/or biochemical parameters could reliably distinguish at risk patients, it may be possible to treat the high risk group with agents to prevent bone loss prior to the development of fractures. An understanding of the mechanism leading to bone loss following OLT is thus required in order to design appropriate treatment schedules.

Histological evidence of increased bone formation at three months following OLT has been found in one study ${ }^{5}$; it was likely that an increase in bone turnover accounted for this finding. Isolated measurements of biochemical markers of bone formation and resorption have revealed an increase in both of these markers following OLT, suggesting an increase in bone turnover rate at this time.$^{5911}$ Elevated levels of parathyroid hormone (PTH) have been observed in the first three months following surgery, which contribute to an increase in bone resorption. ${ }^{11}{ }^{13}$ No study has evaluated serial changes in both BMD and bone biomarkers following OLT. Biochemical markers provide a rapid, non-invasive measurement of bone remodelling activity and can be used to calculate the "uncoupling index", which is the difference or the imbalance between bone formation and resorption. ${ }^{15}$ In an osteoporosis intervention trial using a bisphosphonate, alendronate, investigators showed that early changes in bone biomarkers could predict later changes in BMD. ${ }^{16}$

The aim of this study was to document changes in BMD and bone biomarkers in a group of patients following elective OLT for chronic liver disease, to calculate the uncoupling

Abbreviations used in this paper: $25(\mathrm{OH}) \mathrm{D}$, 25-hydroxyvitamin D; ACR, acute cellular rejection; $\mathrm{BMD}$, bone mineral density; DPD, deoxypyridinoline; FN, femoral neck; LS, lumbar spine; OC, osteocalcin; OLT, orthotopic liver transplantation; PICP, procollagen type 1 carboxy propeptide; $\mathrm{PTH}$, parathyroid hormone; PYD, pyridinoline.
Accepted for publication 21 October 1998 
Table 1 Patient demographics, details of medication, and rejection episodes $(n=12)$

\begin{tabular}{|c|c|c|c|c|c|c|c|}
\hline Patient & Sex & $\begin{array}{l}\text { Age at } \\
\text { OLT } \\
\text { (years) }\end{array}$ & Reason for $O L T$ & $\begin{array}{l}\text { Immunosuppression } \\
\text { schedule }\end{array}$ & $\begin{array}{l}\text { Duration of } \\
\text { steroid treatment } \\
\text { (months) }\end{array}$ & $\begin{array}{l}\text { Episodes of acute } \\
\text { (chronic) rejection }\end{array}$ & $\begin{array}{l}\text { Follow up } \\
\text { (months) }\end{array}$ \\
\hline 1 & M & 53 & PSC & $\begin{array}{l}\text { Triple IM for } 4 / 12 \text { then } \\
\text { dual IM }\end{array}$ & 24 & $1(1)$ & 24 \\
\hline 2 & $\mathrm{~F}$ & 54 & $\mathrm{AIH}$ & Triple IM & 6 & 0 & 18 \\
\hline 3 & $M$ & 48 & PSC & Triple IM & 12 & 2 & 24 \\
\hline 4 & $\mathrm{~F}$ & 48 & Haemochromatosis & Triple IM & 14 & 1 & 24 \\
\hline 5 & $\mathrm{~F}$ & 57 & AIH & Triple IM & 14 & 2 & 18 \\
\hline 6 & $\mathrm{~F}$ & 56 & PBC & Triple IM & 18 & 0 & 24 \\
\hline 7 & M & 56 & PSC & $\begin{array}{l}\text { Triple IM for } 3 / 12 \text { then } \\
\text { dual IM }\end{array}$ & 24 & $2(1)$ & 24 \\
\hline 8 & $\mathrm{~F}$ & 43 & $\mathrm{AIH}$ & $\begin{array}{l}\text { Triple IM for } 3 / 12 \text { then } \\
\text { dual IM }\end{array}$ & 18 & 3 & 18 \\
\hline 9 & $M$ & 57 & Cryptogenic cirrhosis & Triple IM & 6 & 1 & 12 \\
\hline 10 & $\mathrm{~F}$ & 51 & PBC & Triple IM & 10 & 2 & 24 \\
\hline 11 & M & 51 & $\begin{array}{l}\alpha_{1} \text { Antitrypsin } \\
\text { deficiency }\end{array}$ & Triple IM & 12 & 2 & 12 \\
\hline 12 & $\mathrm{~F}$ & 63 & PBC & Triple IM & 6 & 1 & 6 \\
\hline
\end{tabular}

PBC, primary biliary cirrhosis; PSC, primary sclerosing cholangitis; AIH, autoimmune hepatitis; triple IM, immunosuppression with cyclosporin, azathioprine, and steroids; dual IM, immunosuppression with tacrolimus and steroids.

index using biochemical markers, and to assess its value in predicting later changes in bone mass as measured by BMD.

\section{Materials and methods \\ PATIENTS}

Twelve patients (five men and seven women; aged 48-65 years) underwent OLT for a variety of chronic liver diseases (table 1). Each patient received $10 \mathrm{mg} / \mathrm{kg}$ cyclosporin and $2 \mathrm{mg} / \mathrm{kg}$ azathioprine preoperatively and $10 \mathrm{mg} / \mathrm{kg}$ methylprednisolone at induction of anaesthesia and at the anhepatic stage of surgery. After OLT, all patients were maintained on corticosteroids ( 5 $\mathrm{mg} / \mathrm{kg} /$ day, reduced by $5 \mathrm{mg} /$ week), azathioprine $(1 \mathrm{mg} / \mathrm{kg} /$ day), and cyclosporin (dose adjusted according to trough levels obtained by whole blood monoclonal assay) to maintain levels at $150-200 \mu \mathrm{g} / 1$ for the first six months and thereafter at $100-150 \mu \mathrm{g} / \mathrm{l}$. Episodes of acute cellular rejection (ACR) were confirmed histologically and treated with $1 \mathrm{~g}$ methylprednisolone for three consecutive days. Two patients were converted from triple immunosuppression with cyclosporin to tacrolimus therapy with corticosteroids because of the development of chronic rejection (at three and four months) and because of hirsutism due to cyclosporin (at three months). Five women were postmenopausal at the time of the study and two were on low dose corticosteroids preoperatively ( $5 \mathrm{mg} /$ day). None of the patients had a history of bone disease or were on any other medications known to interfere with bone metabolism. Ethical approval was granted by the hospital ethics committee and informed consent obtained from each patient prior to inclusion in the study.

\section{BONE DENSITOMETRY}

Bone mineral density was measured by dual energy $x$ ray absorptiometry using a Hologic QDR 1000 scanner. Measurements were taken at the lumbar vertebrae L1-L4 and at the femoral neck, the precision of measurements at these sites being less than $1 \%$ and $2 \%$ respectively. The results were the composites of L1 to L4 (LS) and the femoral neck $(\mathrm{FN})$, the two areas most prone to fragility fractures. Bone mass was corrected for bone size to calculate $\mathrm{BMD}$ in $\mathrm{g} / \mathrm{cm}^{2}$. The results were compared with peak bone mass for premenopausal women and men and expressed as a T-value, a standard deviation (SD) unit which is calculated as follows:

$$
\text { T-value }=\frac{\mathrm{BMD}_{\text {patient }}-\text { mean } \mathrm{BMD}}{\mathrm{SD}_{\text {reference population }}}
$$

A T-value below -2.5 indicates osteoporosis, a T-value between -2.5 and -1.0 represents osteopenia, and values above -1.0 are considered normal, according to World Health Organisation criteria. ${ }^{17}$ BMD measurements were performed on all patients prior to OLT $(n=12)$ and at three $(n=11)$, six $(n=10)$, nine $(n=8), 12(n=11), 18(n=9)$, and $24(n=6)$ months following surgery.

\section{BIOCHEMICAL MARKERS OF BONE FORMATION} AND RESORPTION

Bone formation was assessed using serum concentration levels of osteocalcin (OC), which is a non-collagenous protein present in bone matrix, and procollagen type 1 carboxy propeptide (PICP), which is the C terminal end of the procollagen molecule released during collagen formation. Bone resorption was assessed using urinary excretion of free pyridinoline (PYD) and deoxypyridinoline (DPD) crosslinks which are present in mature bone and released into the circulation during bone resorption. Vitamin D and calcium status was assessed by measuring serum 25hydroxyvitamin $\mathrm{D}(25(\mathrm{OH}) \mathrm{D})$, total and ionised calcium, phosphate $\left(\mathrm{PO}_{4}\right)$, and $\mathrm{PTH}$ levels. OC was measured by enzyme immunoassay (Bioscience, Ireland) and free PYD and DPD crosslinks were measured by the Metra Biosystems ELISA method. Urinary calcium was measured by atomic absorption spectroscopy and urinary creatinine on a clinical chemical analyser (Cobas Bio) using the Jaffe reaction. Urinary excretion of free pyridinium crosslinks was expressed per mmol of urinary creatinine and performed on a second void two hour urine sample. The precision for measurements of the various biochemical markers was: $6.1 \%$ and $3 \%$ for PICP at concentrations of 78 and $132 \mu \mathrm{g} / 1$ respectively, $5.5 \%$ and $9.5 \%$ for OC at concentrations of 8.1 and $26.2 \mu \mathrm{g} / \mathrm{l}$ respectively, and $7.5 \%$ and $5.3 \%$ for urine free DPD at 24.6 and $122 \mathrm{nmol} / 1$ 
respectively. Serum ionised calcium was measured by ion selective electrode with a radiometer ICA2 analyser. Serum $25(\mathrm{OH}) \mathrm{D}$ was assayed by radioimmunoassay (Incstar Corp., Stillwater, USA) and PTH by immunoradiometric analysis (Allegro-Nichols, San Juan Capistrano, USA). All samples were obtained at 1000 am following an overnight fast and placed on ice and transported to the laboratory immediately following phlebotomy for separation and storage at $-20^{\circ} \mathrm{C}$ until assayed. Biochemical markers were performed on all patients prior to OLT $(n=12)$ and at one $(n=9)$, two $(n=5)$, three $(n=9)$, six $(n=10)$, nine $(n=10), 12(n=11), 18(n=9)$, and $24(n=6)$ months following surgery.

EXPRESSION OF RESULTS OF BIOCHEMICAL

MARKERS

Results of bone resorption markers were measured per mmol of urinary creatinine and bone formation markers in serum as $\mu \mathrm{g} / \mathrm{l}$. Bone formation and resorption markers were then expressed as SD units (T-values) compared with the mean of a sex matched reference population which included premenopausal women $(n=32)$ and men in the $20-45$ year age group $(n=20)$. This system was used to enable a comparison between resorption and formation activities in the patients studied.

STATISTICAL METHODS

Results are given as mean values (SD). Differences between measurements were analysed by the Wilcoxon signed rank test and linear regression analysis was used to examine the correlation between changes in BMD, markers of bone turnover, and demographic variables.

\section{Results}

BONE MINERAL DENSITY

Preoperative BMD T-values at the $\mathrm{LS}$ and $\mathrm{FN}$ were $-1.97(1.28)$ and -1.80 (1.1) respectively; $42 \%$ of patients met the criteria for osteoporosis and $33 \%$ met the criteria for osteopenia. Following OLT, BMD at the LS decreased significantly at three months ( -2.22 (1.23), $\mathrm{p}<0.05)$ compared with baseline. BMD at the LS began to increase after six months, with a significant rise seen between six and 12 months $(-2.50(1.01)$ to $-1.92(0.99), \mathrm{p}<0.01)$ and between 12 and 18 months $(-1.66(-0.97)$, $\mathrm{p}<0.05)$. A slight decrease occurred at 24 months ( $-2.21(0.86), \mathrm{NS}, \mathrm{n}=6)$. BMD continued to improve in eight of the 12 individuals over the time period of the study (fig 1). BMD at the FN decreased significantly from baseline by six months ( -2.27 (0.71), p<0.01) post-OLT. An increase in BMD was noted at this site at nine and 12 months $(-2.16(1.01)$ and $-2.18(0.84)$, NS) with a decrease again noted after this time, at 18 and 24 months $(-2.37(0.77)$ and -2.52 (0.60), NS) (fig 1).

BIOMARKERS

Bone formation

The T-value for OC at baseline was 1.55 (3.4) and decreased immediately post-OLT. OC began to increase again by three months with a significant increase in values between one and

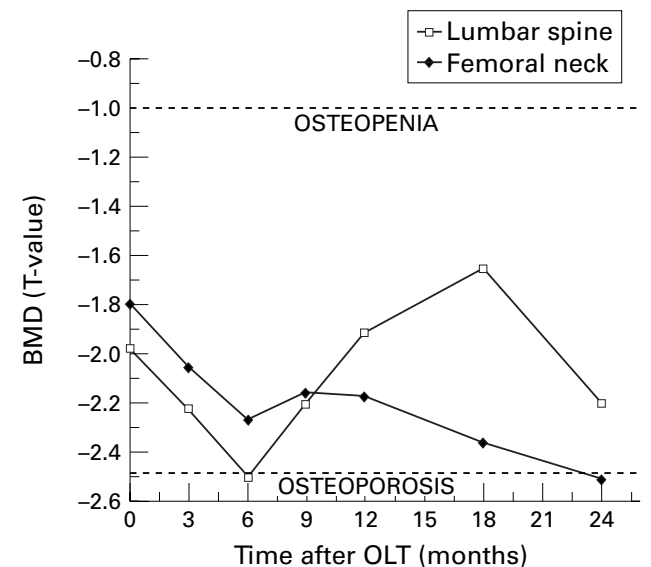

Figure 1 Lumbar spine and femoral neck bone mineral density T-values at baseline and after OLT for 12 patients.

three months ( -0.08 (1.62) versus 3.42 (3.9), $\mathrm{p}<0.05)$ and continued to increase up to nine months (8.80 (6.2), $\mathrm{p}<0.01)$ when it stabilised at a value well above the normal reference range.

At baseline PICP was 2.83 (2.45) and decreased significantly at one month post-OLT (1.03 (1.35), $\mathrm{p}<0.05)$; it began to increase at two months (3.82 (4.43), NS), with a significant increase seen between values at one and three months (3.86 (3.6), p<0.05). PICP stabilised after this time and fell to within the normal reference range by 12 months $(0.94(0.68))$.

\section{Bone resorption}

At baseline DPD was 3.61 (2.24) and began to increase immediately post-OLT, reaching a peak at two months $(6.75(4.0), \mathrm{NS})$; it then stabilised but remained above the normal range (greater than 2.5) for the duration of the study. T-values for both resorption markers, DPD and PYD, correlated significantly with each other at all time points measured $(\mathrm{p}<0.001)$

\section{Uncoupling index (OC minus DPD)}

The difference between T-values for bone formation and resorption indexes (OC DPD) was negative at baseline $(-2.3(3.76)$ ), decreased further in the first two months $(-5.7$ (2.34), NS), then began to rise and was positive by six months $(2.03$ (4.06)), with a significant increase seen between three and nine months

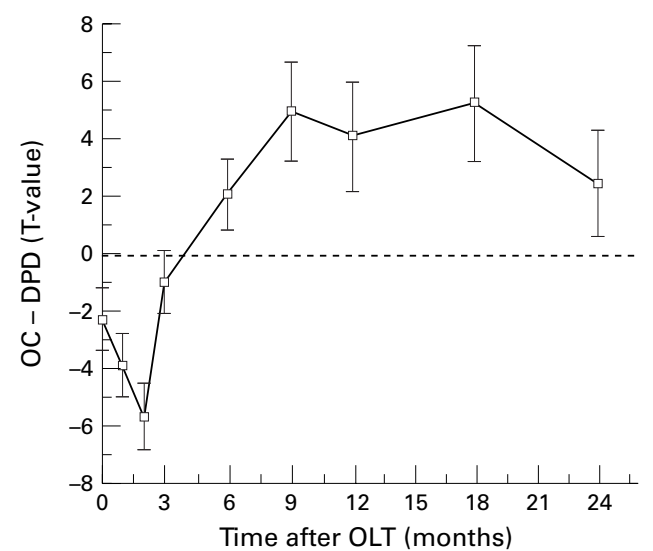

Figure 2 Uncoupling index (OC minus DPD, T-values) at baseline and after OLT for 12 patients. 


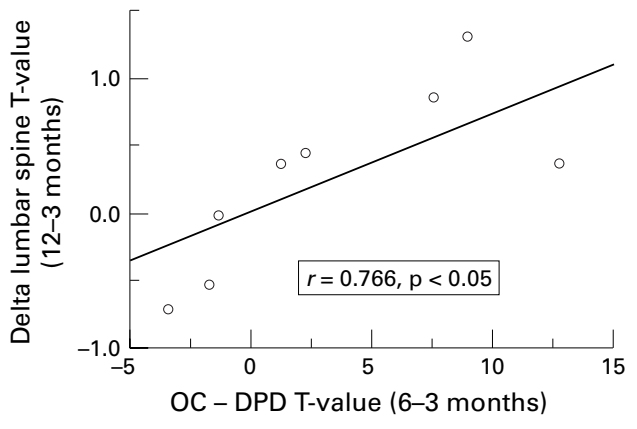

Figure 3 Correlation between changes in uncoupling index (OC minus DPD) at 3-6 months, and lumbar spine bone mineral density at 3-12 months for eight patients $(r=0.766, p<0.05)$.

( -1.01 (3.46) versus 4.91 (5.2), $\mathrm{p}<0.001)$. After this time the uncoupling index stabilised and remained positive for the duration of the study (fig 2). Similar changes were seen for both markers of bone formation (OC and PICP) and both markers of bone resorption (DYD and PYD); therefore only the results of OC - DPD were given to avoid repetition.

There was a significant correlation between the increase in BMD at the LS and FN (between three and 12 months) and the change in the uncoupling index (OC - DPD) between three and six months (LS: $r=0.76, p<0.05$; and $\mathrm{FN}$ : $r=0.62, \mathrm{p}=0.09$ ), for eight patients who had all measurements performed at these times (fig 3). A significant correlation was also found between the increase in BMD and changes in the uncoupling index, expressed as PICP DPD, at similar times (LS: $r=0.74, \mathrm{p}<0.05$ ). The change in the uncoupling index preceded the measured change in BMD and is shown for one patient (fig 4).

VITAMIN D STATUS

Serum 25(OH)D concentrations were below normal (less than $70 \mathrm{nmol} / \mathrm{l}$ ) at baseline (mean 16.6 (8.75)) and increased following OLT but remained below the normal reference range for the study period. Concentrations increased significantly to 46 (17.2) by three months $(\mathrm{p}<0.01)$ and remained relatively stable thereafter. Serum $25(\mathrm{OH}) \mathrm{D}$ concentrations at three months correlated with the increase seen in the BMD both at the LS and FN between baseline

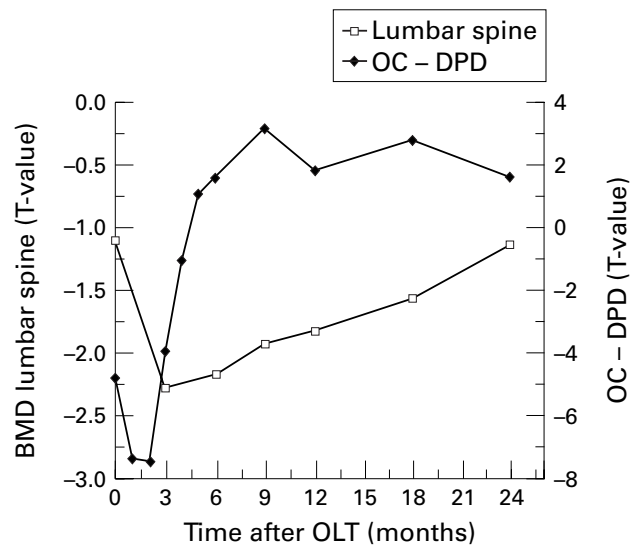

Figure 4 Changes in uncoupling index (OC minus DPD) preceding improvement in lumbar spine bone mineral density, shown here for patient 1. and six months (LS: $r=0.67, \mathrm{p}<0.05$; and $\mathrm{FN}$ : $r=0.71, \mathrm{p}<0.05)$. Serum PTH concentrations were within the normal range (less than 5.5 pmol/1) preoperatively (3.61 (2.7)), but began to increase steadily post-OLT; there was a significant increase seen between concentrations at baseline and three months (3.61 (2.7) versus 4.35 (1.5), $\mathrm{p}<0.05)$ and between concentrations at six and nine months (4.87 (2.0) versus 6.65 (2.2), p<0.05). The mean value for PTH remained above the upper limit of normal at $5.5 \mathrm{pmol} / \mathrm{l}$ for the two years post-OLT.

\section{CLINICAL EVENTS}

Patients spent an average of 27 days (range 16-35) in hospital following surgery. ACR was diagnosed 1.33 times (range 0-3) in each patient during the first three months following OLT. Only one episode of ACR occurred after this time. There was no correlation detected between the change in BMD and the number of episodes of ACR or the length of time spent on corticosteroid medication. The correlation between the change in BMD at the FN from baseline to three months with the number of days spent in hospital approached significance $(r=0.52, \mathrm{p}=0.09)$.

\section{Discussion}

Significant decreases in BMD were seen in the first three to six months following OLT as previously reported. ${ }^{5811}$ An improvement occurred after this time and values were well above baseline at 12 months for the LS. An unexpected fall in BMD was observed at 18 months for the FN and 24 months for the LS for the group, but a continuous improvement was seen in BMD for eight of the 12 patients. Of the four patients who had a decrease in BMD after 18 months (following an initial improvement), two of these had received further corticosteroid therapy for late ACR and a relapse of ulcerative colitis respectively; the latter also underwent surgery for a small bowel obstruction at 22 months (fig 5). The decrease in BMD seen in the other two patients at the LS only remains unexplained; both remained well with normal liver and renal function and were not commenced on any new medications at this time. This deterioration in BMD emphasises the susceptibility of this already compromised patient group to further deterioration even after an initial improvement had been observed in BMD.

Biochemical markers of bone formation and resorption and the calculated uncoupling index indicated that bone resorption exceeded bone formation in this patient group. The greater specificity of the newer markers of bone turnover is generally accepted, as is their use in epidemiological and interventional research studies. The large within subject variability attributed to the markers is a limitation to their usage in individual patients. ${ }^{18}$ The changes observed in the uncoupling index in the first six months correlated with the later changes in BMD and may be a useful technique for identifying at risk groups. Vitamin D status also plays an important role following OLT as shown by the relation between hypovitaminosis 


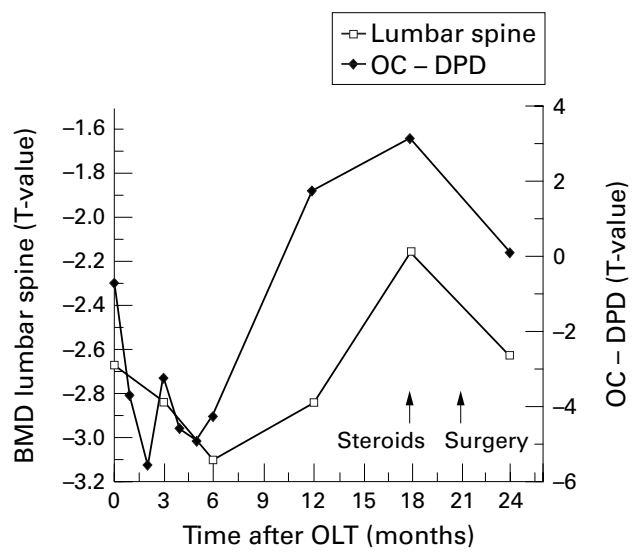

Figure 5 Changes in uncoupling index (OC minus DPD) and LS bone mineral density for patient 3. Arrows indicate clinical events (relapse of ulcerative colitis and surgery for small bowel obstruction) which preceded a fall in both the uncoupling index and BMD seen at 24 months.

$\mathrm{D}$ and bone loss which may reflect compensatory secondary hyperparathyroidism due to inadequate calcium absorption.

Three of the 12 patients were changed from therapy with cyclosporin to tacrolimus during the course of the study because of early chronic rejection $(n=2)$ and hirsutism $(n=1)$. BMD continued to improve in two of these patients and the third had a fall in BMD at 24 months which coincided with corticosteroid therapy for inflammatory bowel disease. It is therefore difficult to conclude whether tacrolimus and cyclosporin had different effects on BMD.

Using non-invasive methods, we have shown that BMD decreased following OLT and is related to a negative uncoupling index. Although this study included a small heterogeneous group of patients the pathogenesis of this bone loss seems to result from an excess of bone resorption relative to formation, as indicated by the negative uncoupling index and its correlation with the loss in BMD at one year. The major risk factors for the development of fractures post-OLT include pre-existing bone loss compounded by the additional bone loss that occurs postoperatively due to immunosuppression and immobility. ${ }^{19}$ The common immunosuppressants - corticosteroids, cyclosporin, and tacrolimus - have been shown to have deleterious effects on bone in both animals and man. ${ }^{20}$ An adverse effect on BMD by cumulative steroid dosage and/or rejection episodes has not been observed in a consistent manner that suggests individual susceptibility of patients to these risk factors. ${ }^{591314}$ Some more recent studies have shown less bone loss than previously following liver transplantation, which may be due to the tendency for lower doses of corticosteroids in immunosuppression regimens, in addition to the earlier referral of fitter patients for liver transplantation and improved medical care following surgery. As seen in this study, even after an initial recovery in BMD, further doses of steroids can have dramatic adverse effects on BMD.

The only randomised controlled treatment trial performed on patients following OLT showed an increase in the vertebral BMD with the antiresorptive agents calcitonin (6.4\%) and bisphosphonates $(8.2 \%)$ compared with a loss of $3.4 \%$ in the untreated group. ${ }^{21} \mathrm{~A}$ nonrandomised trial of cyclical etidronate in combination with vitamin $\mathrm{D}$ and calcium did not improve BMD or fracture rates compared with untreated patients. ${ }^{22}$ The results of this study would suggest that vitamin $\mathrm{D}$ therapy is indicated in all patients post-OLT in view of the notable reduction seen in $25(\mathrm{OH}) \mathrm{D}$ at baseline and the correlation observed with the decrease in BMD. Further controlled trials are required to decide which antiresorptive agent is most effective in patients following OLT.

In conclusion, a decline in BMD occurred in this patient group following OLT and was caused by an excess of bone resorption relative to bone formation, which began to reverse at six months. We have shown early changes in bone biochemical markers which correlate significantly with later changes in BMD. Identification of early changes in bone markers may prove useful in planning therapeutic strategies to prevent bone loss in at risk groups.

We would like to acknowledge the assistance of Barbara Murray and Yvonne McBrinn, biochemists in the Metabolism Laboratory, and Deirdre O'Sullivan, Kate Frazer and Catherine Duane, nurses in the Liver Unit, for their help in collecting samples.

1 Crosbie OM, Freaney R, McKenna MJ, et al. Assessment of fracture risk in patients with chronic liver disease. Ir F Med Sci 1996;165(suppl 2):5.

2 Hay JE. Bone disease in cholestatic liver disease. Gastroenterology 1995;108:276-83.

3 Diamond TH, Steil D, Lunzer M, et al. Hepatic osteodystrophy. Gastroenterology 1989;96:213-21. McCaughan GW, Feller RB. Osteoporosis in chronic liver
disease: pathogenesis, risk factors and management. Dig Dis 1994;12:223-31.

5 McDonald AJ, Dunstan CR, Dilworthi P, et al. Bone loss after liver transplantation. Hepatology 1991;14:613-20.

6 Eastell R, Dickson ER, Hodgson SF, et al. Rates of vertebral bone loss before and after liver transplantation in women with primary biliary cirrhosis. Hepatology 1991;14:296-300.

7 Haagsma EB, Thijn CJP, Post JG, et al. Bone loss after orthotopic liver transplantation. F Hepatol 1988;6:94-100.

8 Porayko MK, Wiesner RH, Hay JE, et al. Bone disease in liver transplant recipients: incidence, timing and risk factors. Transplant Proc 1991;23:1462-5.

9 Meys E, Fontanges E, Fourcade N, et al. Bone loss after liver transplantation. Am f Med 1994;97:445-50.

10 Hay JE. Bone loss in liver transplant recipients. Gastroenterol Clin North Am 1993;22:337-49.

11 Abdelhadi M, Eriksson SAV, Eriksson SL, et al. Bone mineral status in end-stage liver disease and the effect of liver transstatus in end-stage liver disease and the effect of liver
plantation. Scand 7 Gastroenterol 1995;30:1210-15.

12 Hay JE, Dickson ER, Wiesner RH, et al. Long-term effect of orthotopic liver transplantation on the osteopenia of primary biliary cirrhosis. Hepatology 1990;12:838.

13 Compston JE, Greer S, Skingle SJ, et al. Early increase in plasma parathyroid hormone levels following liver transplantation. F Hepatol 1996;25:715-18.

14 Hawkins FG, Leon M, Lopez MB, et al. Bone loss and turnover in patients with liver transplantation. Hepatogastroenterology 1994;41:158-61.

15 Eastell R, Robins SP, Colwell T, et al. Evaluation of bone turnover in type 1 osteoporosis using biochemical markers specific for both bone formation and bone resorption. Osteoporos Int 1993;3:255-60.

16 Garnero P, Shih WJ, Gineyts E, et al. Comparison of new biochemical markers of bone turnover in late postmenopausal osteoporotic women in response to alendronate treatment. F Clin Endocrinol Metab 1994;79:1693-700.

17 Kanis JA, Melton LJ, Christiansen C, et al. The diagnosis of osteoporosis. J Bone Miner Res 1994;9:1137-41.

18 Blumsohn A, Eastell R. The performance and utility of biochemical markers of bone turnover: do we know enough to use them in clinical practice? Ann Clin Biochem 1997;34: 449-59.

19 Epstein S, Shane E, Bilezikian JP. Organ transplantation and osteoporosis. Curr Opin Rheumatol 1995;7:255-61.

20 Katz IA, Epstein S. Posttransplantation bone disease. 7 Bone Miner Res 1992;7:123-6.

21 Valero MA, Loinaz C, Larrodera L, et al. Calcitonin and bisphosphonates treatment in bone loss after liver transbisphosphonates treatment in bone loss a
plantation. Calcif Tissue Int 1995;57:15-19.

22 Riemens SC, Oostdijk A, van Doormaal JJ, et al. Bone loss after liver transplantation is not prevented by cyclical etidafter liver transplantation is not prevented by cyclical etid-
ronate, calcium and alphacalcidol. Osteoporos Int 1996;6: ronate, cal 